This item was submitted to Loughborough's Research Repository by the author.

Items in Figshare are protected by copyright, with all rights reserved, unless otherwise indicated.

\title{
Two new criteria for normal families
}

PLEASE CITE THE PUBLISHED VERSION

LICENCE

CC BY-NC-ND 4.0

REPOSITORY RECORD

Clifford, E.F.. 2019. "Two New Criteria for Normal Families”. figshare. https://hdl.handle.net/2134/477. 


\title{
Two New Criteria for Normal Families
}

\author{
E. F. Clifford
}

\begin{abstract}
Let $a_{0}, \ldots, a_{k-1}$ be analytic functions on a domain $\Omega$. Let $\mathcal{F}$ be a family of meromorphic functions $f$ on $\Omega$ such that $f \neq 0$ and $f^{(k)}+a_{k-1} f^{(k-1)}+\ldots+a_{0} f \neq 0$ on $\Omega$, for all $f \in \mathcal{F}$. Then $\left\{f^{\prime} / f: f \in \mathcal{F}\right\}$ is a normal family. Furthermore, let $a_{0}, \ldots, a_{k-1}$ be meromorphic functions on a domain $\Omega$. Let $\mathcal{F}$ be a family of meromorphic functions $f$ on $\Omega$ such that $f \neq 0, f^{\prime} \neq 0$ and $f^{(k)}+a_{k-1} f^{(k-1)}+\ldots+a_{0} f \neq 0$ on $\Omega$, for all $f \in \mathcal{F}$. Then $\left\{f^{\prime} / f: f \in \mathcal{F}\right\}$ is a normal family. These two new criteria for normal families extend a recent result of Bergweiler and Langley, [1, Corollary 1.1]. ${ }^{1}$
\end{abstract}

Mathematics Subject Classification (1991): 32A19, 32A20, 32A22

Keywords:normal families, meromorphic functions, Nevanlinna theory.

\section{Introduction}

We say that a function is nonvanishing on a domain if it is without zeros there. Since at least as far back as Pólya [13], interest has been shown in determining meromorphic functions $f$ such that $f$ and $f^{(k)}$ are nonvanishing in $\mathbb{C}$ for $k \in \mathbb{N}$. In 1959, Hayman [7] conjectured that the following result would be true.

Theorem A Let $f$ be a meromorphic function in $\mathbb{C}$ and let $k \geq 2$ be an integer. Suppose $f$ and $f^{(k)}$ are nonvanishing in $\mathbb{C}$. Then $f$ has the form $f(z)=e^{a z+b}$ or $f(z)=(a z+b)^{-n}$ where $a, b \in \mathbb{C}, a \neq 0$ and $n \in \mathbb{N}$.

Hayman [7] proved the $k=2$ case for entire functions, and Clunie [3] proved the general case for entire functions. In 1976, Frank [4] proved the $k \geq 3$ case of Theorem A. In 1977, Frank, Hennekemper and Polloczek [6] considered the case where $k \geq 3$ and $f f^{(k)}$ has finitely many zeros. In 1993, Langley [10] proved the $k=2$ case of Theorem A.

Interest has also been shown in determining meromorphic functions $f$ such that $f$ and $L(f)$ are nonvanishing on $\mathbb{C}$, where $L$ is defined by

$$
L(f)=f^{(k)}+a_{k-1} f^{(k-1)}+\ldots+a_{1} f^{\prime}+a_{0} f,
$$

\footnotetext{
${ }^{1}$ Department of Mathematical Sciences, Loughborough University, Leicestershire, LE11 3TU, UK

Email: E.Clifford@lboro.ac.uk

Telephone: +44 (0) 1509222870

Fax: +44 (0) 1509223969
} 
for $k \in \mathbb{N}$. Steinmetz [16], following the work of Frank and Hellerstein [5], proved results for the case where the $a_{j}$ are constants and $k \geq 3$. Brüggemann [2] proved results for the case where the $a_{j}$ are polynomials, not all constant. Langley ([8], [9]) proved results for the case where the $a_{j}$ are rational functions. In this paper, we prove results for the cases where the $a_{j}$ are analytic functions and meromorphic functions.

To see how Theorem A provides a criterion for normal families, we first state the Bloch Principle, noting that this is a heuristic principle and counterexamples do exist, [14].

Bloch Principle A family of meromorphic (analytic) functions which have a common property $\mathcal{P}$ on a domain $\Omega$ will in general be a normal family if $\mathcal{P}$ reduces a meromorphic (analytic) function in $\mathbb{C}$ to a constant.

Next, we note that by Theorem A, if $f$ is an entire function such that $f$ and $f^{(k)}$ are nonvanishing on $\mathbb{C}$ for some $k \geq 2$, then we have that $f(z)=e^{a z+b}$ and so $f^{\prime}(z) / f(z)$ is constant. Then by the Bloch Principle, we see that Theorem A may provide a criterion for normal families. This is in fact the case. Schwick [15] proved this for families of analytic functions, and Bergweiler and Langley [1] proved it for families of meromorphic functions, their results being stated as follows:

Theorem $\mathbf{B}$ ([1]) Let $k \geq 2$ and let $\mathcal{F}$ be a family of functions that are meromorphic in a domain $\Omega$. Suppose that $f$ and $f^{(k)}$ are nonvanishing in $\Omega$, for all $f \in \mathcal{F}$. Then $\left\{f^{\prime} / f: f \in \mathcal{F}\right\}$ is a normal family on $\Omega$.

We include the following example to show that Theorem B does not hold for $k=1$.

Example Let $\mathcal{F}=\left\{f_{n}(z)=\frac{1}{e^{n z}-1}: n \in \mathbb{N}\right\}$. Then $f_{n}(z) \neq 0$ and $f_{n}^{\prime}(z)=-\frac{n e^{n z}}{\left(e^{n z}-1\right)^{2}} \neq 0$ on $\mathbb{C}$, for all $n \in \mathbb{N}$. However, $\left\{f_{n}^{\prime}(z) / f_{n}(z)=-n e^{n z} /\left(e^{n z}-1\right): n \in \mathbb{N}\right\}$ is not a normal family on $\mathbb{C}$ since $f_{n}^{\prime}(0) / f_{n}(0)=\infty$ whereas $f_{n}^{\prime}(x) / f_{n}(x)$

$\rightarrow 0$ for $x \in \mathbb{R}^{-}$, as $n \rightarrow \infty$.

In this paper, we first extend Theorem B to the following result.

Theorem 1.1 Let $k \geq 2$ and let $\mathcal{F}$ be a family of meromorphic functions on a domain $\Omega$. Let $a_{0}, \ldots, a_{k-1}$ be analytic functions on $\Omega$. For each $f \in \mathcal{F}$ define $L(f)$, as in (1), by

$$
L(f)=f^{(k)}+a_{k-1} f^{(k-1)}+\ldots+a_{1} f^{\prime}+a_{0} f .
$$

Suppose that $f$ and $L(f)$ are nonvanishing on $\Omega$ for each $f \in \mathcal{F}$. Then $\left\{f^{\prime} / f: f \in \mathcal{F}\right\}$ is a normal family on $\Omega$.

The following example shows that Theorem 1.1 cannot be extended to the case where $a_{0}, \ldots, a_{k-1}$ are meromorphic functions, in the $k=2$ case.

Example Let $f_{n}(z)=e^{n z} / z$ for $n \in \mathbb{N}$. Set $a_{0}(z)=0$ and $a_{1}(z)=2 / z$. Then for $k=2$, we have

$$
L\left(f_{n}\right)=f_{n}^{(2)}+a_{1}(z) f_{n}^{\prime}(z)+a_{0}(z) f(z)=\frac{n^{2}}{z} e^{n z} .
$$

Thus for all $n \in \mathbb{N}$ we have that $f_{n}(z)$ and $L\left(f_{n}\right)$ are nonvanishing on $\mathbb{C}$. However $\left\{f_{n}^{\prime}(z) / f_{n}(z)=(z-\right.$ $\left.\left.\frac{1}{n}\right) /\left(z \cdot \frac{1}{n}\right): n \in \mathbb{N}\right\}$ is not a normal family on $\mathbb{C}$, since $f_{n}^{\prime}(0) / f_{n}(0)=\infty$, whereas $f_{n}^{\prime}\left(\frac{1}{n}\right) / f_{n}\left(\frac{1}{n}\right)=0$. 
Nevertheless, by including the extra condition that $f^{\prime} \neq 0$ on $\Omega$, for all $f \in \mathcal{F}$, we can extend Theorem 1.1 to the case where $a_{0}, \ldots, a_{k-1}$ are meromorphic functions, as follows:

Theorem 1.2 Let $k \geq 2$ and let $\mathcal{F}$ be a family of meromorphic functions on a domain $\Omega$. Let $a_{0}, \ldots, a_{k-1}$ be meromorphic functions on $\Omega$. For each $f \in \mathcal{F}$ define $L(f)$, as in (1), by

$$
L(f)=f^{(k)}+a_{k-1} f^{(k-1)}+\ldots+a_{1} f^{\prime}+a_{0} f .
$$

Suppose that $f, f^{\prime}$ and $L(f)$ are nonvanishing on $\Omega$ for each $f \in \mathcal{F}$. Then $\mathcal{G}=\left\{f^{\prime} / f: f \in \mathcal{F}\right\}$ is a normal family on $\Omega$.

\section{Proof of Theorem 1.1}

We follow a similar method of proof to that used by Bergweiler and Langley in their proof of Theorem B.

First, we define differential polynomials $\Psi_{k}(F)$ for $k \in \mathbb{N}$ by

$$
\Psi_{1}(F)=F, \quad \Psi_{k+1}(F)=F \Psi_{k}(F)+\left(\Psi_{k}(F)\right)^{\prime} .
$$

The link between these operators and nonvanishing derivatives is given by the following lemma from [1], which can be easily proved by induction.

Lemma 2.1 ([1]) Let $f$ be meromorphic on a domain $\Omega$ and let $F=f^{\prime} / f$. Then for each $k \in \mathbb{N}$ we have $\Psi_{k}(F)=f^{(k)} / f$.

Next, let $a_{0}, \ldots, a_{k-1}$ be analytic functions on a domain $\Omega$, and define differential polynomials $\Lambda_{k}(F)$ for $k \in \mathbb{N}$ by

$$
\Lambda_{k}(F)=\Psi_{k}(F)+a_{k-1} \Psi_{k-1}(F)+\ldots+a_{1} \Psi_{1}(F)+a_{0} .
$$

We then use the following theorem, which is an extension of [1, Theorem 1.3].

Theorem 2.2 Let $k \geq 2$ and let $\mathcal{F}$ be a family of functions meromorphic in a domain $\Omega$. Let $a_{0}, \ldots, a_{k-1}$ be analytic functions on $\Omega$. For $F \in \mathcal{F}$ define $\Lambda_{k}(F)$, as in (3), by

$$
\Lambda_{k}(F)=\Psi_{k}(F)+a_{k-1} \Psi_{k-1}(F)+\ldots+a_{1} \Psi_{1}(F)+a_{0} .
$$

Suppose that there exists $\delta \in(0,1]$ such that the following conditions hold for all $F \in \mathcal{F}$ :

(i) $\Lambda_{k}(F)$ has no zeros.

(ii) if $a$ is a simple pole of $F$ then $|\operatorname{Res}(F, a)-j| \geq \delta$ for $j \in\{0,1, \ldots, k-1\}$.

(iii) if $c \in \Omega$ and $R>0$ with $D(c, R) \subset \Omega$, if $D(c, \delta R)$ contains two poles of $F$, counting multiplicities, and if $D(c, R) \backslash D(c, \delta R)$ contains no poles of $F$, then

$$
\left|\sum_{a \in D(c, \delta R)} \operatorname{Res}(F, a)-(k-1)\right| \geq \delta .
$$


Then $\mathcal{F}$ is a normal family.

We prove this theorem in $\S 4$, and provide an example there to show that Theorem 2.2 cannot be extended to the case where $a_{0}, \ldots, a_{k-1}$ are meromorphic functions.

Finally, we can deduce Theorem 1.1 from Theorem 2.2 as follows. First we note that conditions $(i i)$ and (iii) of Theorem 2.2 are satisfied if we have that $\operatorname{Re}(\operatorname{Res}(F, a)) \leq-\delta$ for all poles $a$ of $F$. This is the case, in particular, if $F=f^{\prime} / f$ for some nonvanishing meromorphic function $f$. Then by (3) and since $\Psi_{k}(F)=f^{(k)} / f$ for $k \in \mathbb{N}$ by Lemma 2.1, we have that Theorem 1.1 follows.

\section{Proof of Theorem 1.2}

Proof Let $\left\{\alpha_{j}: j \in J\right\}$ be the set of poles of $a_{0}, \ldots, a_{k-1}$ in $\Omega$. By Theorem 1.1, the result is true in the case where $a_{0}, \ldots, a_{k-1}$ are analytic functions, and so it is sufficient to prove that $\mathcal{G}$ is normal at the $\alpha_{j}$, for $j \in J$.

Suppose there exists $\alpha_{j}$ such that $\mathcal{G}$ is not normal at $\alpha_{j}$. Choose $\delta>0$ such that the punctured disc $\Omega_{j}=\left\{z: 0<\left|z-\alpha_{j}\right|<\delta\right\}$ is contained in $\Omega$, and such that $\Omega_{j}$ does not contain any poles of $a_{0}, \ldots, a_{k-1}$. Then we have that there exists a sequence $\left(F_{n}\right)$ in $\mathcal{G}$, where $F_{n}=f_{n}^{\prime} / f_{n}$ for some $f_{n} \in \mathcal{F}$, such that $\left(f_{n}^{\prime} / f_{n}\right)$ has no subsequence that converges locally uniformly on $\Omega_{j} \cup\left\{\alpha_{j}\right\}$. However, since $a_{0}, \ldots, a_{k-1}$ are analytic in $\Omega_{j}$, we have by Theorem 1.1 that $\mathcal{G}$ is normal on $\Omega_{j}$. Then there exists a subsequence of $\left(f_{n}^{\prime} / f_{n}\right)$, denoted $\left(f_{n}^{\prime} / f_{n}\right)$ without loss of generality, which converges uniformly on compact sub-regions of $\Omega_{j}$, either to a meromorphic limit $\phi$, or identically to $\infty$. Then there are two cases.

Case 1. $\left(f_{n}^{\prime} / f_{n}\right)$ converges uniformly to a meromorphic limit $\phi$ on compact sub-regions of $\Omega_{j}$.

We note first that for $n \in \mathbb{N}$, since $f_{n} \neq 0$ on $\Omega$, the poles of $f_{n}^{\prime} / f_{n}$ can only arise at poles of $f_{n}^{\prime}$, and therefore only at poles of $f_{n}$. We note that for the remainder of this proof, we refer to the poles of $f_{n}^{\prime} / f_{n}$ only as the poles of $f_{n}$. We note also that $f_{n}^{\prime} / f_{n}$ has no zeros on $\Omega$ since $f_{n}^{\prime} \neq 0$ there.

Let $\Gamma$ be a circular contour in $\Omega_{j}$ which goes once anti-clockwise around $\alpha_{j}$ and which does not pass through any poles of $\phi$. Since $\phi$ is a meromorphic limit, $\Gamma$ lies in the interior of a closed annulus $\mathcal{A}$ on which $\phi$ has no poles. Further, since $\left(f_{n}^{\prime} / f_{n}\right)$ converges uniformly to $\phi$ on compact sub-regions of $\Omega_{j}$, we have that there exists $n_{0} \in \mathbb{N}$ such that $f_{n}$ has no poles on $\mathcal{A}$ for $n \geq n_{0}$.

Let $\Omega_{\Gamma}$ be the domain enclosed by $\Gamma$. By the Argument principle, and since each $f_{n}$ has no zeros in $\Omega$, we have that for each $n \in \mathbb{N}$,

$$
\int_{\Gamma}\left(\frac{f_{n}^{\prime}}{f_{n}}\right)(z) d z=-2 \pi i q_{n}
$$

where $q_{n}$ is the number of poles of $f_{n}$ in $\Omega_{\Gamma}$, counting multiplicities. Then since,

$$
\lim _{n \rightarrow \infty} \int_{\Gamma}\left(\frac{f_{n}^{\prime}}{f_{n}}\right)(z) d z=\int_{\Gamma} \phi(z) d z=\lambda
$$

for some $\lambda \in \mathbb{C}$, we have that there exists $n_{1} \in \mathbb{N}$ such that $n_{1} \geq n_{0}$ and such that $\int_{\Gamma}\left(f_{n}^{\prime} / f_{n}\right)(z) d z$ is constant for $n \geq n_{1}$. Then by (4), we must have that for $n \geq n_{1}$, the $f_{n}$ have the same number of poles, say $q$, in $\Omega_{\Gamma}$. 
We list the poles by $\gamma_{n, 1}, \ldots, \gamma_{n, q}$ repeating according to multiplicity. Then we can write for $n \geq n_{1}$,

$$
\left(\frac{f_{n}^{\prime}}{f_{n}}\right)(z)=\sum_{l=1}^{q}\left(-\frac{1}{z-\gamma_{n, l}}\right)+\psi_{n}(z)
$$

where $\psi_{n}$ is an analytic function on $\Omega_{\Gamma} \cup \mathcal{A}$.

Next, we show that the $\psi_{n}$ are uniformly bounded on $\Omega_{\Gamma}$. We note first that $\gamma_{n, 1}, \ldots, \gamma_{n, q}$ are not in the closed annulus $\mathcal{A}$, and so $|\phi(z)| \leq C_{1}$ and $\left|z-\gamma_{n, l}\right| \geq c_{1}$ for some positive constants $C_{1}$ and $c_{1}$, for $z \in \Gamma$. Since $\left(f_{n}^{\prime} / f_{n}\right)$ converges uniformly to $\phi$ on $\Gamma$, there exists $n_{2} \in \mathbb{N}$ such that $n_{2} \geq n_{1}$ and such that, on $\Gamma$, $\left|\left(f_{n}^{\prime} / f_{n}\right)(z)\right| \leq C_{1}+1$ for $n \geq n_{2}$. Since $\left|z-\gamma_{n, l}\right| \geq c_{1}$, we have that $\left|\sum_{l=1}^{q}\left(-\frac{1}{z-\gamma_{n, l}}\right)\right| \leq q / c_{1}$. Then we have that, on $\Gamma$,

$$
\left|\psi_{n}(z)\right| \leq\left|\left(\frac{f_{n}^{\prime}}{f_{n}}\right)(z)\right|+\left|\sum_{l=1}^{q}\left(-\frac{1}{z-\gamma_{n, l}}\right)\right| \leq C_{1}+1+q / c_{1},
$$

for $n \geq n_{2}$. Therefore the $\psi_{n}$ are uniformly bounded on $\Gamma$. By the maximum principle, the $\psi_{n}$ are uniformly bounded on $\Omega_{\Gamma}$.

Choose a subsequence of $\left(\gamma_{n, l}\right)$, denoted $\left(\gamma_{n, l}\right)$ without loss of generality, such that $\gamma_{n, l} \rightarrow \gamma_{l}$ as $n \rightarrow \infty$ for $l=1, \ldots, q$. Then there are two subcases, depending on whether some of $\gamma_{1}, \ldots, \gamma_{q}$ are equal to $\alpha_{j}$.

Case 1.1. Some of $\gamma_{1}, \ldots, \gamma_{q}$ are equal to $\alpha_{j}$.

Rearrange $\gamma_{1}, \ldots, \gamma_{q}$ so that $\gamma_{1}, \ldots, \gamma_{p}$ are equal to $\alpha_{j}$ and $\gamma_{p+1}, \ldots, \gamma_{q}$ are not equal to $\alpha_{j}$, for some $p \in\{1, \ldots, q\}$. Then by (5), we have for $n \geq n_{2}$,

$$
\left(\frac{f_{n}^{\prime}}{f_{n}}\right)(z)=\sum_{l=1}^{p}\left(-\frac{1}{z-\gamma_{n, l}}\right)+\sum_{l=p+1}^{q}\left(-\frac{1}{z-\gamma_{n, l}}\right)+\psi_{n}(z) .
$$

Next, since $\gamma_{n, p+1}, \ldots, \gamma_{n, q}$ tend to $\gamma_{p+1}, \ldots, \gamma_{q}$ as $n \rightarrow \infty$, and $\gamma_{p+1}, \ldots, \gamma_{q}$ are not equal to $\alpha_{j}$, we can choose $\delta_{1}>0$ such that $B\left(\alpha_{j}, 3 \delta_{1}\right)$ is contained in $\Omega_{\Gamma} \backslash \mathcal{A}$ and does not contain $\gamma_{p+1}, \ldots, \gamma_{q}$. Then there exists $n_{3} \in \mathbb{N}$ such that $n_{3} \geq n_{2}$ and such that, for $n \geq n_{3}$ and $z \in B\left(\alpha_{j}, \delta_{1}\right)$, we have $\left|z-\gamma_{n, l}\right| \geq \delta_{1}$ for $l=p+1, \ldots, q$. Then for each $n \geq n_{3}$, we have $\left|\sum_{l=p+1}^{q}\left(-\frac{1}{z-\gamma_{n, l}}\right)\right| \leq(q-p) / \delta_{1}$ and so $\sum_{l=p+1}^{q}\left(-\frac{1}{z-\gamma_{n, l}}\right)$ is uniformly bounded and analytic in $B\left(\alpha_{j}, \delta_{1}\right)$. Then since the $\psi_{n}$ are uniformly bounded on $\Omega_{\Gamma}$, there exists a large positive constant $M$ such that for $n \geq n_{3}$,

$$
\left|\sum_{l=p+1}^{q}\left(-\frac{1}{z-\gamma_{n, l}}\right)+\psi_{n}(z)\right| \leq M
$$

on $B\left(\alpha_{j}, \delta_{1}\right)$.

Now choose $\delta_{2}>0$ such that $\delta_{2} / \delta_{1}$ is small, and consider the circle $S\left(\alpha_{j}, \delta_{2}\right)=\left\{z:\left|z-\alpha_{j}\right|=\delta_{2}\right\}$. We have that $\gamma_{n, 1}, \ldots, \gamma_{n, p}$ each tend to $\alpha_{j}$ as $n \rightarrow \infty$, and so each $\left|-\frac{1}{z-\gamma_{n, l}}\right|$ is large on $S\left(\alpha_{j}, \delta_{2}\right)$ for $l=1, \ldots, p$. Then, in particular, we have that

$$
\left|\sum_{l=1}^{p}-\frac{1}{z-\gamma_{n, l}}\right| \rightarrow\left|\sum_{l=1}^{p}-\frac{1}{z-\gamma_{l}}\right|=\left|-\frac{p}{z-\alpha_{j}}\right|=\frac{p}{\delta_{2}}>2 M,
$$


as $n \rightarrow \infty$, for a suitable choice of $\delta_{2}$.

Then by (6) and (7), there exists $n_{4} \in \mathbb{N}$ such that $n_{4} \geq n_{3}$ and such that $f_{n}^{\prime} / f_{n}$ is large on $S\left(\alpha_{j}, \delta_{2}\right)$ for $n \geq n_{4}$, and thus $f_{n} / f_{n}^{\prime}$ is small on $S\left(\alpha_{j}, \delta_{2}\right)$ for $n \geq n_{4}$. Next, we know that each $f_{n} / f_{n}^{\prime}$ is analytic in $\Omega$ since $f_{n}^{\prime} \neq 0$ on $\Omega$. Then for $n \geq n_{4}$, by the maximum principle and since $f_{n} / f_{n}^{\prime}$ is small on $S\left(\alpha_{j}, \delta_{2}\right)$, each $f_{n} / f_{n}^{\prime}$ is small on $B\left(\alpha_{j}, \delta_{2}\right)$. Then $\left(f_{n} / f_{n}^{\prime}\right)$ is a uniformly bounded sequence of analytic functions on $B\left(\alpha_{j}, \delta_{2}\right)$, and by the Montel-Vitali theorem, we have that $\left(f_{n} / f_{n}^{\prime}\right)$ is normal on $B\left(\alpha_{j}, \delta_{2}\right)$. Therefore $\left(f_{n}^{\prime} / f_{n}\right)$ is normal on $B\left(\alpha_{j}, \delta_{2}\right)$, and thus, in particular, $\left(f_{n}^{\prime} / f_{n}\right)$ is normal at $\alpha_{j}$. This is a contradiction.

Case 1.2. $\alpha_{j} \neq \gamma_{l}$ for all $l=1, \ldots, q$.

Then we can choose $\delta_{3}>0$ such that $B\left(\alpha_{j}, 3 \delta_{3}\right)$ is contained in $\Omega_{\Gamma} \backslash \mathcal{A}$, and does not contain $\gamma_{1}, \ldots, \gamma_{q}$. Then there exists $n_{5} \in \mathbb{N}$ such that $n_{5} \geq n_{3}$ and such that, for $n \geq n_{5}$ and $z \in B\left(\alpha_{j}, \delta_{3}\right)$, we have $\left|z-\gamma_{n, l}\right| \geq \delta_{3}$ for $l=1, \ldots, q$. Then $\left|\sum_{l=1}^{q}-\frac{1}{z-\gamma_{n, l}}\right| \leq \frac{q}{\delta_{3}}$, and since $\sum_{l=1}^{q}\left(-\frac{1}{z_{n}-\gamma_{n, l}}\right)$ is uniformly bounded and analytic in $B\left(\alpha_{j}, \delta_{3}\right)$ and since the $\psi_{n}$ are uniformly bounded on $\Omega_{\Gamma}$, we have that $(7)$ holds with $p=0$, on $B\left(\alpha_{j}, \delta_{3}\right)$.

Therefore, $\left(f_{n}^{\prime} / f_{n}\right)$ is a uniformly bounded sequence of analytic functions on $B\left(\alpha_{j}, \delta_{3}\right)$. Then by the MontelVitali theorem, we have that $\left(f_{n}^{\prime} / f_{n}\right)$ is normal on $B\left(\alpha_{j}, \delta_{3}\right)$, and thus, in particular, $\left(f_{n}^{\prime} / f_{n}\right)$ is normal at $\alpha_{j}$. This is a contradiction.

Case 2. $\left(f_{n}^{\prime} / f_{n}\right)$ converges identically to $\infty$ on $\Omega_{j}$.

Then we have that $\left(f_{n} / f_{n}^{\prime}\right)$ converges identically to 0 on $\Omega_{j}$. We note that for each $n$, we have that $f_{n} / f_{n}^{\prime}$ is analytic in $\Omega_{j} \cup\left\{\alpha_{j}\right\}$ since $f_{n}^{\prime} \neq 0$ on $\Omega$. Then by the maximum principle, we have that $\left(\left(f_{n} / f_{n}^{\prime}\right)\left(\alpha_{j}\right)\right)$ converges to 0 , and so $\left(f_{n}^{\prime} / f_{n}\right)$ converges identically to $\infty$ on $\Omega_{j} \cup\left\{\alpha_{j}\right\}$. Therefore $\left(f_{n}^{\prime} / f_{n}\right)$ is normal on $\Omega_{j} \cup\left\{\alpha_{j}\right\}$. This is a contradiction. Therefore $\mathcal{G}$ is a normal family.

\section{Proof of Theorem 2.2}

We need several lemmas for the proof of Theorem 2.2. The first assertion in the following lemma is proved in [1], and the second is an extension which follows immediately.

Lemma 4.1 ([1]) Let $k \geq 2$ be an integer. Let $y$ be meromorphic on a domain $\Omega$, such that if a is a simple pole of $y$ then $\operatorname{Res}(y, a) \notin\{1, \ldots, k-1\}$. Let $n \in \mathbb{N}$ be such that $n \leq k$. If $y$ has a pole at a of multiplicity $m$ then $\Psi_{n}(y)$ has a pole at a of multiplicity $n m$, and $\Lambda_{n}(y)$ has a pole at a of multiplicity $n m$, where $\Lambda_{n}(y)$ is defined as in (3) by

$$
\Lambda_{n}(y)=\Psi_{n}(y)+a_{n-1} \Psi_{n-1}(y)+\ldots+a_{1} \Psi_{1}(y)+a_{0}
$$

where $a_{0}, \ldots, a_{n-1}$ are analytic functions on $\Omega$.

We need the following theorems of Bergweiler and Langley.

Theorem 4.2 ([1]) Let $k \geq 3$ be an integer, and let $F$ be meromorphic and nonconstant in the plane and satisfy both of the following conditions:

(i) $\Psi_{k}(F)$ has no zeros. 
(ii) if $a$ is a simple pole of $F$ then $\operatorname{Res}(F, a) \notin\{1, \ldots, k-1\}$.

Then $F$ has the form

$$
F(z)=\frac{(k-1) z+\alpha}{z^{2}+\beta z+\gamma},
$$

or

$$
F(z)=\frac{1}{\alpha z+\beta} .
$$

Here $\alpha, \beta, \gamma \in \mathbb{C}$ with $\alpha \neq 0$ in (9).

Conversely, if $F$ has the form (8) or (9), and if (ii) holds, then $\Psi_{k}(F)$ has no zeros. If $F$ has the form (8) or (9), but (ii) does not hold, then $\Psi_{k}(F) \equiv 0$.

Theorem 4.3 ([1]) Let $F$ be meromorphic and nonconstant in the plane, such that

(i) $\Psi_{2}(F)=F^{\prime}+F^{2}$ has no zeros.

(ii) if $a$ is a simple pole of $F$, then $\operatorname{Res}(F, a) \neq 1$.

(iii) there exists $\delta>0$ such that if $a$ is a simple pole of $F$ then $|\operatorname{Res}(F, a)| \geq \delta$.

Then either $F$ has the form (8) with $k=2$, or the form (9).

We also need the following lemma of Pang and Zalcman, see [1], [11] and [12].

Lemma $4.4([\mathbf{1 2}])$ Let $\mathcal{F}$ be a family of functions meromorphic on the unit disc $D(0,1)$. Suppose that there exists $\delta>0$ such that if $f \in \mathcal{F}$ has a simple pole a, then $|\operatorname{Res}(f, a)| \geq \delta$. Then if $\mathcal{F}$ is not normal, there exist a number $r \in(0,1)$, points $z_{n} \in D(0, r)$, functions $F_{n} \in \mathcal{F}$ and positive numbers $\rho_{n}$ tending to zero such that

$$
\rho_{n} F_{n}\left(z_{n}+\rho_{n} z\right) \rightarrow F(z)
$$

locally uniformly in $\mathbb{C}$, where $F$ is a nonconstant meromorphic function on $\mathbb{C}$ such that $F^{\sharp}(z) \leq F^{\sharp}(0)=$ $1+1 / \delta$ for all $z \in \mathbb{C}$, in which $F^{\sharp}$ denotes the spherical derivative.

The proof of Theorem 2.2 will involve rescaling, as in Lemma 4.4, and so we need the following results which can be easily proved.

Lemma 4.5 Let $F$ and $g$ be functions such that

$$
g(z)=\rho F(a+\rho z)
$$

where $\rho>0, a \in \mathbb{C}$. Then the following statements are true:

(a) If $g$ has a pole $a t b$, then $F$ has a pole at $a+\rho b$, and $\operatorname{Res}(g, b)=\operatorname{Res}(F, a+\rho b)$.

(b) For each $j \in \mathbb{N}$, we have

$$
\Psi_{j}(g)(z)=\rho^{j} \Psi_{j}(F)(a+\rho z)
$$

where $\Psi_{j}$ is defined as in (2). 
(c) If $a_{0}, \ldots, a_{k-1}$ are analytic functions and

$$
\Lambda_{k}(F)=\Psi_{k}(F)+a_{k-1} \Psi_{k-1}(F)+\ldots+a_{0}
$$

as defined by (3), then

$$
\Lambda_{k}(F)(a+\rho z)=\rho^{-k}\left[\Psi_{k}(g)(z)+\sum_{j=1}^{k-1} a_{j}(a+\rho z) \rho^{k-j} \Psi_{j}(g)(z)+\rho^{k} a_{0}(a+\rho z)\right] .
$$

We proceed to the proof of Theorem 2.2. We note that it uses essentially the same methods as $[1$, Theorem $1.3]$.

Proof Since normality is a local property we can assume, without loss of generality, that $\Omega$ is a disc. We can assume also that the $a_{j}$ are bounded on $\Omega$ since they are analytic functions. Using a linear change of variables $g(z)=\rho F(a+\rho z)$, for suitable choice of $\rho>0$ and $a \in \mathbb{C}$, we may assume that $\Omega$ is the open unit disc $D(0,1)$ since Lemma 4.5 (a) shows that the residues of $g(z)$ are unaltered, and Lemma 4.5 (c) shows that if $\Lambda_{k}(F)$ is nonvanishing then $\tilde{\Lambda}_{k}(g)$ is nonvanishing, where the coefficients of $\tilde{\Lambda}_{k}$ are given by (10). Suppose now that $\mathcal{F}$ is not normal. Then by condition $(i i)$ of Theorem 2.2, with $j=0$, we can apply Lemma 4.4. Let $r, z_{n}, F_{n}, \rho_{n}$ and $F$ be as in Lemma 4.4, so that,

$$
g_{n}(z)=\rho_{n} F_{n}\left(z_{n}+\rho_{n} z\right) \rightarrow F(z)
$$

locally uniformly in $\mathbb{C}$ as $n \rightarrow \infty$.

Let $a$ be a simple pole of $F$. Then, by Hurwitz' Theorem, if $n$ is sufficiently large, $g_{n}$ has a simple pole at $a_{n}$ with $a_{n} \rightarrow a$ as $n \rightarrow \infty$. By Lemma 4.5 (a), $z_{n}+\rho_{n} a_{n}$ is a simple pole of $F_{n}$ with $\operatorname{Res}\left(F_{n}, z_{n}+\rho_{n} a_{n}\right)=$ $\operatorname{Res}\left(g_{n}, a_{n}\right)$. Hence, with $\delta \in(0,1]$, we deduce from condition $(i i)$ of Theorem 2.2 that $\left|\operatorname{Res}\left(g_{n}, a_{n}\right)-j\right| \geq \delta$ for $j \in\{0,1, \ldots, k-1\}$. Then we have that $|\operatorname{Res}(F, a)-j| \geq \delta$ for $j \in\{0,1, \ldots, k-1\}$. And so, by Lemma 4.1 , every pole of $F$ is a pole of $\Psi_{k}(F)$.

Next, by Lemma 4.5 (c), we have

$$
\Lambda_{k}\left(F_{n}\right)\left(z_{n}+\rho_{n} z\right)=\rho_{n}^{-k}\left[\Psi_{k}\left(g_{n}\right)(z)+\sum_{j=1}^{k-1} a_{j}\left(z_{n}+\rho_{n} z\right) \rho_{n}^{k-j} \Psi_{j}\left(g_{n}\right)(z)+\rho_{n}^{k} a_{0}\left(z_{n}+\rho_{n} z\right)\right] .
$$

By condition $(i)$ of Theorem 2.2, this has no zeros. Hence,

$$
\tilde{\Lambda}_{k}\left(g_{n}\right)(z)=\Psi_{k}\left(g_{n}(z)\right)+\sum_{j=1}^{k-1} a_{j}\left(z_{n}+\rho_{n} z\right) \rho_{n}^{k-j} \Psi_{j}\left(g_{n}(z)\right)+\rho_{n}^{k} a_{0}\left(z_{n}+\rho_{n} z\right)
$$

has no zeros, since $\rho_{n}$ is a sequence of positive numbers. We know by (2) that $\Psi_{j}\left(g_{n}\right)$ is a linear combination of products of $g_{n}$ and its derivatives. Let $\mathbb{E}$ be the set of poles of $F$. Then, by the Weierstrass Theorem, we have that

$$
\tilde{\Lambda}_{k}\left(g_{n}\right)(z) \rightarrow \Psi_{k}(F)(z)
$$

as $n \rightarrow \infty$, locally uniformly on $\mathbb{C} \backslash \mathbb{E}$, since $\rho_{n} \rightarrow 0$ and the $a_{j}$ are bounded. By Hurwitz' Theorem, either $\Psi_{k}(F) \equiv 0$ or $\Psi_{k}(F)$ has no zeros on $\mathbb{C} \backslash \mathbb{E}$. In the latter case, we deduce that $\Psi_{k}(F)$ has no zeros at all since every pole of $F$ is a pole of $\Psi_{k}(F)$. 
Case 1. $\Psi_{k}(F) \equiv 0$.

Since $|\operatorname{Res}(F, a)-j| \geq \delta$ for $j \in\{0,1, \ldots, k-1\}$, if $a$ is a simple pole of $F$, we deduce from Lemma 4.1 that $F$ has no poles. Thus $F$ is entire and so is the function $f$ defined by setting $f(z)=\exp \left(\int_{0}^{z} F(t) d t\right)$. Then $F=f^{\prime} / f$ and thus $f^{(k)} / f=\Psi_{k}(F) \equiv 0$ by Lemma 2.1. Hence $f$ is a polynomial. This implies that $f$ is constant. Hence $F \equiv 0$, which is a contradiction, since $F$ is nonconstant.

Case 2. $\Psi_{k}(F)$ has no zeros.

It follows from Theorem 4.2 for $k \geq 3$ and from Theorem 4.3 for $k=2$ that $F$ has the form (8) or (9). Suppose first that $F$ has the form (9). Then $1 /|\alpha|=|\operatorname{Res}(F,-\beta / \alpha)| \geq \delta$ so that $|\alpha| \leq 1 / \delta$. On the other hand, $|\alpha| \geq|\alpha| /\left(1+|\beta|^{2}\right)=F^{\sharp}(0)=1+1 / \delta$. This is a contradiction.

Suppose second that $F$ has the form (8) but is not of the form (9). Then $F$ has two poles, counting multiplicity. We also observe that if $F$ is of the form (8), then

$$
\sum_{a \in F^{-1}(\{\infty\})} \operatorname{Res}(F, a)=k-1
$$

by the Residue Theorem. Next, choose $R>0$ such that these poles are contained in $D(0, \delta R)$. Since $F$ has no other poles we deduce from Hurwitz' theorem that for $n$ sufficiently large, $g_{n}$ has two poles in $D(0, \delta R)$, but no poles in $D(0, R) \backslash D(0, \delta R)$. Thus $F_{n}$ has two poles in $D\left(z_{n}, \delta \rho_{n} R\right)$, but no poles in $D\left(z_{n}, \rho_{n} R\right) \backslash D\left(z_{n}, \delta \rho_{n} R\right)$. By Lemma 4.5 (a) and condition (iii) of Theorem 2.2, we deduce that

$$
\left|\sum_{a \in D(0, \delta R)} \operatorname{Res}\left(g_{n}, a\right)-(k-1)\right|=\left|\sum_{a \in D\left(z_{n}, \delta \rho_{n} R\right)} \operatorname{Res}\left(F_{n}, a\right)-(k-1)\right| \geq \delta .
$$

But this gives

$$
\left|\sum_{a \in D(0, \delta R)} \operatorname{Res}(F, a)-(k-1)\right| \geq \delta
$$

which contradicts (11). This completes the proof of Theorem 2.2.

Finally, we use an example to show that Theorem 2.2 cannot be extended to the case where $a_{0}, \ldots, a_{k-1}$ are meromorphic functions.

Example Let $k=2$ and let $\mathcal{F}$ be the family of meromorphic functions $\left\{F_{n}(z)=\frac{1}{n z^{3}}: n \in \mathbb{N}\right\}$. Let $a_{0}(z)=0$ and $a_{1}(z)=3 / z$. Then $a_{0}$ and $a_{1}$ are meromorphic functions and for $F_{n} \in \mathcal{F}$ we have

$$
\begin{aligned}
\Lambda_{2}\left(F_{n}\right)(z) & =\Psi_{2}\left(F_{n}\right)(z)+a_{1}(z) \Psi_{1}\left(F_{n}\right)(z)+a_{0}(z) \\
& =\frac{1}{n^{2} z^{6}}-\frac{3}{n z^{4}}+\frac{3}{z} \frac{1}{n z^{3}}=\frac{1}{n^{2} z^{6}} .
\end{aligned}
$$

This has no zeros, and so condition $(i)$ is satisfied.

Also, each $F \in \mathcal{F}$ has a triple pole at $z=0$, and no other poles. Thus conditions $(i i)$ and (iii) are trivially satisfied. Thus all the conditions of Theorem 2.2 are satisfied except for the analyticity of the $a_{j}$. However $\mathcal{F}$ is not a normal family since $F_{n}(0)=\infty$ while $F_{n}(z)=\frac{1}{n z^{3}} \rightarrow 0$ as $n \rightarrow \infty$ for $z \in \mathbb{R}^{+}$. Therefore Theorem 2.2 cannot be extended to the case where $a_{0}, \ldots, a_{k-1}$ are meromorphic functions, in the $k=2$ case. Similar counterexamples can be constructed in the general case.

It is interesting to note here, with regard to Theorem 1.2 , that although the $F_{n}$ in this example have no zeros, they cannot be written in the form $F_{n}=f_{n}^{\prime} / f_{n}$ where $f_{n}$ is a nonvanishing meromorphic function. 
ACKNowledgements: This research has been done as part of a PhD thesis at the University of Nottingham. The author gratefully acknowledges the advice and support of Prof J. K. Langley. The author also thanks the referee(s) for their useful suggestions.

\section{References}

[1] Walter Bergweiler and J. K. Langley, Nonvanishing derivatives and normal families, J. Anal. Math. 91 (2003), 353-367. MR 2037414

[2] F. Brüggemann, Proof of a conjecture of Frank and Langley concerning zeros of meromorphic functions and linear differential polynomials, Analysis 12 (1992), 5-30.

[3] J. Clunie, On integral and meromorphic functions, J. London Math. Soc. 37 (1962), 17-27. MR 26 \#1456

[4] Günter Frank, Eine Vermutung von Hayman über Nullstellen meromorpher Funktionen, Math. Z. 149 (1976), no. 1, 29-36. MR 54 \#10601

[5] Günter Frank and Simon Hellerstein, On the meromorphic solutions of nonhomogeneous linear differential equations with polynomial coefficients, Proc. London Math. Soc. (3) 53 (1986), no. 3, 407-428. MR 88k:30032

[6] Günter Frank, Wilhelm Hennekemper, and Gisela Polloczek, Über die Nullstellen meromorpher Funktionen und deren Ableitungen, Math. Ann. 225 (1977), no. 2, 145-154. MR 55 \#3257

[7] W. K. Hayman, Picard values of meromorphic functions and their derivatives, Ann. of Math. (2) 70 (1959), 9-42. MR $22 \# 1675$

[8] J. K. Langley, The Tsuji characteristic and zeros of linear differential polynomials, Analysis 9 (1989), no. 3, 269-282. MR 90k:30056

[9] _ An application of the Tsuji characteristic, J. Fac. Sci. Univ. Tokyo Sect. IA Math. 38 (1991), no. 2, 299-318. MR 92m:30060

[10] _ Proof of a conjecture of Hayman concerning $f$ and $f^{\prime \prime}$, J. London Math. Soc. (2) 48 (1993), no. 3, 500-514. MR 94k:30075

[11] Xuecheng Pang, Shared values and normal families, Analysis (Munich) 22 (2002), no. 2, 175-182. MR 2003h:30043

[12] Xuecheng Pang and Lawrence Zalcman, Normal families and shared values, Bull. London Math. Soc. 32 (2000), no. 3, 325-331. MR 2001e:30059

[13] G. Pólya, Über die Nullstellen sukzessiver Derivierten, Math. Z. 12 (1922), 36-60.

[14] Lee A. Rubel, Four counterexamples to Bloch's principle, Proc. Amer. Math. Soc. 98 (1986), no. 2, 257-260. MR 87i:30064

[15] Wilhelm Schwick, Normality criteria for families of meromorphic functions, J. Analyse Math. 52 (1989), 241-289. MR 90k:30061 
[16] Norbert Steinmetz, On the zeros of $\left(f^{(p)}+a_{p-1} f^{(p-1)}+\cdots+a_{0} f\right) f$, Analysis 7 (1987), no. 3-4, 375-389. MR 89e:34059 